\title{
Genotype-Phenotype Correlations in Neurofibromatosis and Their Potential Clinical \\ Use
}

Chetan Bettegowda, MD, PhD, Meena Upadhayaya, PhD, D. Gareth Evans, MD, AeRang Kim, MD, PhD, Dimitrios Mathios, MD, and Clemens O. Hanemann, MD, PhD, on behalf of the REiNS International Collaboration

Neurology ${ }^{\circledR}$ 2021;97:S91-S98. doi:10.1212/WNL.0000000000012436

\author{
Correspondence \\ Dr. Hanemann \\ Oliver.Hanemann@ \\ plymouth.ac.uk
}

\begin{abstract}
\section{Objective}

Because clinically validated biomarkers for neurofibromatosis 1 (NF1) and neurofibromatosis 2 (NF2) have not been identified, we aimed to determine whether genotype-phenotype correlations are useful in clinical trials in NF1 and NF2.

\section{Methods}

The Response Evaluation in Neurofibromatosis and Schwannomatosis (REiNS) Biomarker Group first performed a systematic literature search and reviewed existing data on genetic biomarkers in NF1 and NF2 and in in malignant peripheral nerve sheath tumors. The group then met during a series of consensus meetings to develop a joint report.

\section{Results}

We found that in NF2, the genetic severity score is clearly of potential clinical use. In NF1, despite over 3,000 constitutional variants having been described in the NF1 gene, only 4 actionable genotype-phenotype correlations exist. The diagnosis and treatment decision of these tumors should ideally include histopathology and compilation of some of the genetic markers.
\end{abstract}

\section{Conclusion}

We summarized emerging clinical use of genotype-phenotype correlations in neurofibromatosis.

From Johns Hopkins University School of Medicine (C.B., D.M.), Baltimore, MD; Division Cancer and Genetics (M.U.), Cardiff University; Genomic Medicine (D.G.E.), University of Manchester, UK; Center for Cancer and Blood Disorders (A.K.), Children's National Hospital, Washington, DC; and Faculty of Health, Medicine, Dentistry and Health Sciences (C.O.H.), Institute of Translational and Stratified Medicine, University of Plymouth, UK.

Go to Neurology.org/N for full disclosures. Funding information and disclosures deemed relevant by the authors, if any, are provided at the end of the article. 


\section{Glossary}

ANF = atypical neurofibroma; FSNF = familial spinal neurofibromatosis; LOVD = Leiden Open Variation Database; MPNST = malignant peripheral nerve sheath tumor; NAHR = nonallelic homologous recombination; NF1 = neurofibromatosis type 1; NF2 = neurofibromatosis type 2; ODS = Optimum Discrimination Score; PH = pleckstrin homology; PTA = pure tone average; REiNS = Response Evaluation in Neurofibromatosis and Schwannomatosis; UAB = University of Alabama.

The Response Evaluation in Neurofibromatosis and Schwannomatosis (REiNS) Biomarker Group reviews biomarkers in blood, urine, and tissue for neurofibromatosis 1 (NF1) and neurofibromatosis 2 (NF2) and schwannomatosis. Our previous publication $^{1}$ defined biomarker needs in NF1, NF2, and schwannomatosis and concentrated on recommendations for protein biomarkers. Here we explore the clinical usefulness of genotype-phenotype relation in NF1 and NF2. We concentrate on constitutive mutation in NF1 and NF2 and discuss somatic mutations in malignant peripheral nerve sheath tumors (MPNSTs). We explore whether mutation analysis could be used to stratify outcomes in clinical trials. In addition, we discuss whether some trials should focus selectively on certain genotypes. In the future, mutation analysis may help to select for gene therapy approaches. We also touch on potential MPNST biomarkers resulting from somatic mutations.

\section{Methods}

The Biomarker Group first performed a systematic literature search and reviewed existing data on genetic biomarkers in NF1 and NF2. The group then met during a series of consensus meetings to (1) nominate individual members to summarize the literature in their areas of expertise and assure data comparison between studies and (2) develop a joint report. This report was the circulated to patient representatives and REiNS Director Council for comments.

\section{Results}

\section{Neurofibromatosis 2}

The hallmark of NF2 is the development of bilateral, frequently multifocal eighth cranial nerve (vestibular) schwannomas leading to hearing loss and balance disturbance. ${ }^{2,3}$ Schwannomas occur on other cranial, spinal, and peripheral nerve roots and there are also characteristic plaque-like intracutaneous schwannomas. ${ }^{4}$ Meningiomas, which are mostly fibroblastic or atypical, occur throughout the neuroaxis and are associated with increased mortality. ${ }^{5,6}$ Intraspinal lowgrade ependymomas also occur and are usually indolent despite their appearance on MRI. ${ }^{7}$ In the United Kingdom, large population-based estimates of birth incidence for NF2 showed that between 1 in 25-33,000 people are born with a pathogenic variant in the NF2 gene. ${ }^{8}$ Just over $50 \%$ of NF2affected individuals present with no family history, and about a third to half of these are mosaic for NF2, as the mutation is only present in a subset of cells, indicating that the initial mutation occurred during embryogenesis. ${ }^{9}$

NF2 is caused by loss of function mutations in the NF2 tumor suppressor gene on chromosome 22q. ${ }^{4}$ Mutations in NF2 follow the 2-hit hypothesis, where the first constitutional hit can be different types of mutations from point mutations to large rearrangements, and the second hit in the tumor is frequently loss of heterozygosity. Large studies have determined genotype-phenotype correlations with truncating pathogenic variants (nonsense and frameshift) conferring more severe disease courses than missense mutations, splice site mutations, or large deletions. ${ }^{10,11}$ In addition, the position of the mutation correlates with mutations in the $3^{\prime}$ end of the gene (exons 14/15) being associated with fewer meningiomas ${ }^{12}$ and lower mortality. Mosaic-affected individuals have a milder form of NF2, consistent with fewer cells carrying the pathogenic variant., ${ }^{9,13}$

A study in 142 UK patients led to the suggestion of a genetic severity score using the following genotype-phenotype correlations: ${ }^{13}$

1. Type 1: Mild: mosaic for mutations only found in tumour, not blood

2. Type 2a: Mild: missense variants, exon 1 and 13/14 truncating, splicing $7-15$, mosaic for variants except in $2 b$ in blood

3. Type 2b: Moderately severe: large deletions, splicing variants exons 1-6, mosaic for truncating variants (exons 2-13) in blood

4. Type 3: Severe: truncating mutations exons $2-13$

Type 3 variants are associated with very early mortality with almost no one living beyond 60 years. ${ }^{6}$ These are associated with frequent childhood onset ${ }^{14}$ and high frequency of meningiomas. Type $2 b$ are intermediate with significantly better survival than type $3,{ }^{6,11}$ but more severe than type $2 \mathrm{a}$. The classification also accounts for later and milder disease caused by mosaic variants that are not found in blood analysis. ${ }^{9,13}$ Accordingly, there is a statistically significant but weak correlation of the genetic severity score with quality of life and number of interventions. ${ }^{13}$

A follow-up study showed that genetic severity is a significant predictor of hearing outcomes including Optimum Discrimination Scores (ODS), hearing classification, and maximum annual pure tone average (PTA) deterioration. Median age of serviceable hearing varied from 32 to 80 years depending on 
genetic severity. ${ }^{15}$ The authors use a mild genetic severity score in counseling the patient in clinic. Nonetheless, age of individuals with NF2 needs to be taken into account, especially in those with presymptomatic testing.

\section{Neurofibromatosis 1}

Identification of a specific $N F 1$ variant cannot generally predict the progression or outcome of the disease in a patient with NF1, even within a family. It is important to note that the development of many NF1-related tumors, including that of MPNST, one of the most lethal manifestations of NF1, is a 2-hit phenomenon. Phenotype is regulated by multiple factors including agedependent manifestations, the timing and number of second hits in specific cells, allelic and nonallelic heterogeneity, cellular heterogeneity, epigenetics, modifying loci, and environmental and stochastic factors. It is the interplay of all these factors that determines a specific phenotype. Identification of a genotypephenotype correlation for a particular constitutional variant or variant type aids in the clinical management and genetic counseling of patients. However, although more than 3,197 different constitutional NF1 pathogenic variants have been identified (hgmd.cf.c.uk/), only 4 clinically confirmed genotype-phenotype correlations have been reported, relevant to $10 \%-15 \%$ of the NF1 population.

\section{Germline Genetic Modifications Contributing to the Genotype-Phenotype Correlation in NF1}

\section{NF1 p.Met992del}

This genotype-phenotype correlation was described in 2007 involving the in-frame deletion of codon 992: p.Met992del. ${ }^{16}$ Patients with this variant have a milder phenotype primarily comprising café-au-lait spots and skinfold freckling, and lack cutaneous and visible plexiform neurofibromas, which are the hallmark features of NF1 (see also D. Wallis in this issue). The study cohort in this international study included 21 patients (14 familial and 7 sporadic) and 26 affected relatives. The other clinical features described in this cohort of p.Met992del patients include learning problems (17\%), pectus anomalies (16\%), short stature $(11 \%)$, scoliosis $(10 \%)$, pulmonary stenosis $(9 \%)$, macrocephaly (9\%), and symptomatic spinal neurofibroma (2\%). A subsequent larger study confirmed these findings, and also failed to find external visible plexiform or cutaneous neurofibromas. ${ }^{17}$ Unlike the previous study, $4.8 \%$ of individuals were found to have nonoptic pathway tumors, but they were mostly low-grade and asymptomatic. A higher proportion (38.8\%) had cognitive impairment/learning disabilities, compared to the $17 \%$ reported. ${ }^{16}$ The overall prevalence of lipomas in individuals with p.Met922del in both studies combined was 5.5\%. The molecular mechanism associated with this mutation remains unknown. The frequency of the p.Met992del variant in NF1 mutationpositive unrelated individuals in the NF1 Leiden Open Variation Database (LOVD) is $0.78 \%(27 / 3,442)$ and in the University of Alabama (UAB) cohort is reported to be $0.88 \%(74 / 8,400) .{ }^{17,18}$

This mild phenotypic spectrum overlaps with the clinical features observed in Legius syndrome, which is caused by pathogenic variants in SPRED $1 .{ }^{19}$ However, patients with Legius syndrome are distinct from those with NF1 in not having Lisch nodules.

\section{NF1 p.Arg1809}

This genotype-phenotype correlation was first reported in $2015,{ }^{20}$ involving a missense change at codon 1809, an arginine residue that is highly conserved and located in the pleckstrin homology $(\mathrm{PH})$ domain of neurofibromin. Six unrelated patients with NF1 with p.Arg1809Cys due to NF1 c.5425C $>\mathrm{T}$ exhibited café-au-lait spots and freckling, macrocephaly, thoracic abnormalities, reduced growth, and learning problems. Notably similar to p.Met992del, these patients did not have discrete cutaneous, spinal, or plexiform neurofibromas, optic pathway gliomas, other malignancies, or skeletal abnormalities. These findings were confirmed by a multicenter comprehensive study. ${ }^{21}$ In approximately $25 \%$ of the individuals, Noonan-like features could be found. Pulmonic stenosis and short stature were significantly more prevalent compared with classic cohorts $(p<0.0001)$. In over $50 \%$ of patients, developmental delays or learning disabilities were reported. Melanocytes cultured from a café-au-lait spot in a patient with segmental NF1 showed 2 different somatic NF1 mutations, p.Arg1809Cys and a multiexon deletion, providing genetic evidence that p.Arg1809Cys is a loss-of-function mutation in the melanocytes and causes a pigmentary phenotype. Constitutional missense variants at p.Arg1809 are reported in $\sim 1.23 \%$ of unrelated NF1 probands: $0.87 \%$ (30/ $3,442)$ in the NF1 LOVD and $1.23 \%$ in the UAB cohort. ${ }^{18,21}$

We suggest that patients/families with the above-named mutations should not be included in natural history studies or clinical trials investigating plexiform neurofibromas.

\section{NF1 Microdeletion}

About $4.7 \%-11 \%$ of patients with NF1 have a so-called "microdeletion" of 14 protein coding genes including NF1 and 4 microRNA genes. Three different size NF1 microdeletions have been reported. ${ }^{22}$ The commonest type of NF1 microdeletion, accounting for $70 \%-80 \%$ of such cases, is type 1 , which spans $1.4 \mathrm{Mb}$ and is estimated to occur with a frequency of 1 in $60,000 .^{22}$

Most type 1 NF1 microdeletions are caused by interchromosomal nonallelic homologous recombination (NAHR) during maternal meiosis. The NAHR is facilitated by the presence of recurrent breakpoints in low-copy repeats NF1-REPa and NF1-REPc.

Type 2 NF1 microdeletions encompass $1.2 \mathrm{Mb}$ and are associated with hemizygosity of 13 protein coding genes, including $L R R C 37 B$. They are caused by mitotic rather than meiotic NAHR and hence are associated with somatic mosaicism and a less severe phenotype. ${ }^{23}$ The breakpoints of type 2 deletions map to SUZ12 and its pseudogene SUZ12P1, which flank NF1-REPc and NF1-REPa. At least $10 \%$ of NF1 microdeletions are type 2 . 
Type 3 NF1 microdeletion encompasses $1.0 \mathrm{Mb}$ and accounts for $1 \%-4 \%$ of all patients with large NF1 deletions. In contrast to type 1 microdeletions, type $3 \mathrm{~s}$ do not include the 5 functional genes CRLF3, ATAD5, TEFM, ADAP2, and RNF135. Only 10 patients with NF1 with $1.0 \mathrm{Mb}$ deletion have been reported. Cognitive impairment was observed in only $50 \%$ (4/8 patients). Type 3 microdeletions are mediated by NAHR between NF1-REPb and NF1-REPc leading to hemizygosity of 9 protein-coding genes.

Type 4 microdeletions are unusual in that they are not associated with recurrent breakpoints and thus have a variable number of genes in the deleted region. Type 4 microdeletions can be both constitutional and postzygotic. It is estimated that these constitute $8 \%-10 \%$ of all large deletions.

Patients with NF1 with type 1, 1.4 Mb deletions, exhibit a more severe phenotype. ${ }^{22,24,25}$ These patients have increased numbers of cutaneous, subcutaneous, plexiform, and spinal neurofibromas as compared with the general NF1 population. They also have an extremely high burden of internal neurofibromas. They have fourfold increased risk of MPNST. Codeletion of SUZ12 or EED gene in addition to NF1 further increases MPNST risk and hemizygosity of ATAd5, COPRS, UTP6, and RNF135 also contribute to increased tumor risk. Complete loss of PRC2 (SUZ12, EED) function in plexiform neurofibroma derived from patients with microdeletion is important for malignant transformation to MPNSTs. In addition, these patients have dysmorphic facial features, are tall for their age, and exhibit other features of overgrowth, such as large hands and feet, hyperflexibility of joints, skeletal abnormalities, and muscular hypotonia. These patients are associated with impaired cognitive development and increased cardiovascular anomalies as compared with the general NF1 population. Loss of RNF135 in the microdeleted region is considered to be the cause of the dysmorphic facial features and overgrowth. ${ }^{26}$

Somatic mosaicism for type 1 microdeletions is rare: only 3 such patients have been reported; 2 of these patients exhibited general manifestation of NF1 and the third had segmental NF1. All 3 had a milder phenotype than that seen in typical type 1 microdeletion. ${ }^{27}$ Overall clinical severity of the patients with microdeletion is determined by the size of the deletion and somatic mosaicism.

\section{Missense Mutations in NF1 Codons 844-848}

The fourth genotype-phenotype correlation is with missense mutations affecting 1 of the 5 codons $844-848$ in the cysteine-serine rich (CSR) domain, which is associated with a severe phenotype. ${ }^{32}$ This study included 129 unrelated probands and 33 affected relatives. These patients have a high prevalence of plexiform or spinal neurofibromas, symptomatic and asymptomatic optic pathway gliomas, malignant neoplasms, and skeletal abnormalities.

This severe phenotype was observed in $75 \%$ of adult NF1affected individuals with these variants in codons 844-848, clearly demonstrating that missense mutations outside the GTPase-activating protein-related domain (GRD) can be associated with a severe clinical presentation. A total of $25 \%$ of patients with NF1 with such variants do not have a typical severe phenotype. Missense and single amino acid deletions can be less detrimental as they alter only a discrete region of protein and perhaps affect protein function in a more precise manner.

Focusing on the recurrent and highly conserved missense variants may provide more predictive markers. Four clear genotype-phenotype correlations have been identified so far, offering biomarkers for clinical management and genetic counseling. Notably, each of the genotype-phenotype correlations affects only a small percentage of individuals with NF1: $5.9 \%$ with microdeletions, $0.78 \%$ with p.Met992del, 0.9\%-1.2\% with p.Arg1809 missense variants, and 1.6\% with missense variants at codons $844-848 .{ }^{18}$ Taken together, therefore, approximately $10 \%$ of patients with NF1 can be counseled more specifically about the likely progression of certain aspects of their disease. Patients and families with p.Met992del and p.Arg1809 missense variants should likely not be included in natural history or clinical studies investigating plexiform neurofibromas as these manifestations do not occur in this small subset. However, one has to take into account that plexiform neurofibromas are congenital and frequently detected by imaging, especially when whole-body MRI is done routinely. We are just beginning to unravel the relationship between specific variants or types of variants and clinical features of NF1 after nearly 30 years of study. Availability of a large number of clinically and molecularly well-characterized patients with NF1 contributed by multiple genetic centers will pave the way for future genotype-phenotype correlations.

Other NF1 genotype-phenotype correlations that have not been confirmed in larger datasets are described below.

\section{Missense or Splice-Site NF1 Mutations: FSNF}

Spinal tumors that develop in patients with classic NF1 usually occur in small numbers and only affect one region of the spine, with most symptomatic tumors situated below the cervical level. The NF1 constitutional variant spectrum associated with such patients is typical of that observed in the general NF1 population. In contrast, patients with familial spinal neurofibromatosis (FSNF) present with multiple bilateral spinal tumors involving large regions of the spine, frequently causing symptoms resulting from cervical spinal cord compression. Despite these symptomatic tumors, these patients exhibit few if any other NF1 clinical features. A number of FSNF families have been reported and their constitutional NF1 variants studied. ${ }^{28-32}$

The risk of having FSNF vs classical NF1 was significantly increased in individuals harboring missense or splice site variants. $^{30,32}$

\section{Breast Cancer}

In a cohort of 78 patients with NF1 with breast cancer, it was highly significant that no cases were observed with either 
partial or whole gene deletions $(p=0.014)$, suggesting that patients with microdeletion are not at increased risk of breast cancer (hazard ratio 0.11 ). ${ }^{33}$ While no overall correlations were observed between other variant types and the risk of breast cancer, 45 (64.3\%) of the 70 different variants observed were enriched, i.e., were observed more frequently than expected, with $p$ values $0.001-0.049$ and associated hazard ratios 6.4-83. In addition, a higher proportion of nonsense variants were observed in association with breast cancer over the age of 50 years, and in $90 \%(10 / 11)$ of those with missense variants and known age at onset, breast cancer occurred under 50 years $(p=0.041)$. These findings require confirmation in a larger independent cohort and individual clinicians will need to decide on actionability.

\section{Somatic Genetic Changes and Epigenetic Modifications Contributing to Phenotypic Variation in Patients With NF1}

The progression from a normal Schwann cell to an MPNST is a phenomenon that requires multiple genetic and epigenetic changes to be orchestrated under a supportive microenvironment. ${ }^{3437}$ In the majority of cases, a patient with NF1 will initially develop a plexiform neurofibroma that over time will transform to an MPNST. ${ }^{38}$ The second hit in the process of MPNST formation in patients with NF1 is somatic mutations acquired at the level of haploinsufficient (NF1 \pm ) Schwann cells that lead to additional deletion or activation of key genes important in cancer-related pathways. $^{35}$

In the majority of the cases, neurofibromas are distinguishable from MPNST that exhibit increased cellularity, increased mitosis, cytologic atypia, and sometimes necrosis. However, there are cases that show mixed features of lower grade and higher grade and they are difficult to classify. ${ }^{39}$ This group of neurofibromas is collectively called atypical neurofibroma (ANF).$^{40}$ Of these, some will remain benign over time, whereas others will progress to MPNST within a few years from initial diagnosis. A recent classification motif groups the latter under the term atypical neurofibromatous neoplasms of uncertain biologic potential (ANNUBP) $)^{41}$ to indicate the greater risk these ANF have for transformation to MPNST.

\section{Somatic Mutations in ANF and MPNST}

Somatic mutation burden and genomic instability in ANF is comparatively low, with only $N F 1, C D K N 2 A / B$, and, to a lesser extent, SMARCA2 mutated in the tumors. SUZ12, EED, or TP53, which are frequently inactivated in MPNST, are not mutated in ANF. Comparing unmatched neurofibromas vs MPNST from the pooled NF1 population demonstrates loss of $C D K N 2 A / B$ appears to be the main genetic event that in addition to NF1 inactivation leads to premalignancy. The transition to MPNST coincides with a rise in genomic instability; inactivation of PRC2 complex genes such as SUZ12, EED, or KDM2B ${ }^{37}$; and copynumber gains of cell cycle and pluripotency genes. ${ }^{42,43}$

A longitudinal analysis of patients with NF1 from diagnosis with a neurofibroma to the transformation to an MPNST has the advantages to analyze the spatial and temporal mutations of neurofibromas in these patients. Hirbe et al. ${ }^{44}$ performed whole exome sequencing in a patient with NF1 who had progression of a lesion from plexiform neurofibroma to MPNST and metastasis and identified an increasing number of cells with somatic inactivation of NF1 during progression of the disease. They identified loss of 1 copy of TP53 in MPNST and its metastasis but not in the plexiform neurofibroma.

\section{DNA Methylation/Histone Modifications in the Progression From Neurofibroma to MPNST}

Multiple studies demonstrate that the transformation from plexiform neurofibroma to MPNST is an epigenetic phenomenon. Specifically, loss of SUZ12, EED, or KDM2B genes in MPNST inactivates the PRC2 pathway responsible for methylation of the lysine 27 of histone $\mathrm{H} 3$, leading to hyperactivation of multiple key cancer-related and developmental pathways. ${ }^{35,36}$ Development of MPNST in patients with NF1 may be a 3-hit phenomenon where NF1 is lost with SUZ12 as part of the microdeletion syndromes as a first hit and consequently somatic NF1 loss as a second hit with a final hit being the loss of the remaining final SUZ12 copy leading to complete inactivation of the PRC2 complex. ${ }^{35}$ Immunohistochemistry of MPNST demonstrates decreased levels of $5 \mathrm{mC}, 5 \mathrm{hmC}$, and H3K27me3 in MPNST compared to plexiform neurofibromas and dermal neurofibromas. ${ }^{45}$ Hypermethylation of CDKN2A, WT1, and S100B is frequent in MPNST compared to neurofibromas in human samples. ${ }^{46}$ Methylome profiling of Schwann cells, neurofibroma, and MPNST from patients with NF1 using methylated DNA immunoprecipitation sequencing (MeDIP-seq) technology showed that there was no significant global hypomethylation in MPNST compared to neurofibromas or Schwann cells ${ }^{47}$ in contrast to what has been reported for other tumor types. ${ }^{48}$ However, satellite repeats showed a highly significant directional difference in DNA methylation, suggesting these repeats represent the main target for hypomethylation in MPNST. ${ }^{47}$ The functional significance of this pattern of hypomethylation in the repeat regions of MPNST genome remains unclear. In addition, a key number of genes in MPNST are identified as being hypermethylated, driving a suppressive effect of RNA expression of these genes. For example, the $\mathrm{CpG}$ island of the promoter region of SOX1O and CDKN2A were highly hypermethylated in MPNST compared to neurofibromas or Schwann cells, leading to decreased gene expression.

\section{Application of Genetic Data in Diagnostics and Prognostication of MPNST}

Despite many efforts and the significant increase in the amount of genetic information known about MPNST, there is still no blood-based or tumor-based genetic marker that can distinguish with certainty the transition of a neurofibroma to MPNST. As a result, the diagnosis of MPNST is made based on careful analysis of the whole tissue given for histopathology and compilation of some genetic markers.

One promising marker that can help in the diagnosis of MPNST is the assessment of $\mathrm{H} 3 \mathrm{~K} 27 \mathrm{me} 3$ by immunohistochemistry. Loss 
of $\mathrm{H} 3 \mathrm{~K} 27 \mathrm{me} 3$ points to the diagnosis of MPNST, but the presence of $\mathrm{H} 3 \mathrm{~K} 27 \mathrm{me} 3$ does not exclude the diagnosis of MPNST. ${ }^{45}$ Schwann cell markers (S100, Sox10) are often lost in MPNST. Loss of CDKN2A as mentioned above differentiates plexiform neurofibromas from ANF and MPNST but may not differentiate the 2 entities. TP53 intense positivity points to MPNST.

There are few specific genetic aberrations identified as prognostic markers for survival in MPNST. RASSF1 promoter methylation was associated with decreased survival in patients with NF1associated MPNST, but this difference in survival was not noted in sporadic MPNST. ${ }^{46}$ Hypomethylation of the MPNST specimens was associated with increased RNA expression of the RASSF1 gene. The RASSF1 gene is important in regulation of microtubule formation and it is therefore conceivable that decreased expression of the gene can lead to genomic instability that is associated with higher grade lesions. ATRX protein expression is an NF1-specific prognostic marker of survival in MPNST, but does not appear to be correlated in sporadic cases. ${ }^{49}$ ATRX is a gene that regulates telomere lengthening and its loss leads to immortalization of tumor cells. It can affect the PRC2 complex to regulate methylation of histones leading to regulation of key developmental and cancer-related pathways. ATRX mutations have a well-established role in gliomagenesis and progression of glial tumors and many other malignancies.

\section{Discussion}

Genotype-phenotype correlations in humans are complex, as phenotype is neither homogeneous nor perceptible. With the advent of next-generation sequencing, the vast genetic variations reflected in the form of single nucleotide polymorphisms, polymorphisms, frameshift insertions and deletions, copy number variants, and triplet repeats may be good predictors.

In NF2, the genetic severity score is clearly of potential clinical use. Clinical trials will need to adjust for genetic severity. Ideally, any randomization should stratify by age and genetic severity category. Early phase trials should probably be confined to type $2 b$ and 3 .

In NF1, despite over 3,000 constitutional variants having been described in the NF1 gene, only 4 actionable genotype-phenotype correlations exist. A diagnosis of NF1 can be confidently made in a majority of patients by using the clinical diagnostic criteria supported by molecular tests. Although information on NF1 germline mutations can be easily achieved, we lack sufficient knowledge of the regulatory and unlinked genetic factors. As few variants can predict the severity and progression of the disease, many women from families with NF1 do not opt for prenatal testing because the severity of disease cannot be accurately predicted on an individual basis. Therefore, additional biomarkers for genotype-phenotype relationships are needed. With increasing knowledge of MPNST pathogenesis, the diagnosis and treatment decision of these tumors include histopathology and compilation of some of the genetic markers.

The paucity of well-characterized genotype-phenotype correlations may be due to the marked genetic heterogeneity seen in patients with NF1, lack of variant clustering, and that a majority of constitutional variants are private. Clinical manifestations are often age-dependent, therefore, it is imperative that children be included in future studies. Other hampering factors include observed intrafamily and interfamily clinical variability, mosaicism in the founder member, multiple modifying loci, and environmental factors. In addition, without functional analyses, one cannot be absolutely confident about the pathogenicity of a nonrecurrent missense variant. Comprehensive clinical details are required for each patient, but in a busy clinic this can be a challenging task for a physician. The Human Phenome Project, which requires phenotype data to be recorded in a systematic way, as has been done in Decipher ${ }^{50}$ and the 100,000 Genomes Project, will further aid the analysis of genotype-phenotype correlations.

Human Phenotype Ontology ${ }^{51}$ allows machine searchable description of phenotype. Integrating data on DNA variants into knowledge networks and reasoning them with artificial intelligence could help define deep genotype. Artificial intelligence could also be useful in predicting genotype-phenotype correlation by deep phenotype of the clinical information from the electronic health records and integrating that with genomic data. Deep learning methodologies have also been employed to predict sequence specificity of DNA and RNA binding proteins.

Extensive research into the genetic and epigenetic analysis of NF1-related tumors shows the significance of DNA methylation and histone modifications, as well as the accumulation of somatic mutations through the progression of benign to malignant tumors, as important factors contributing to the development of phenotypic features that cannot otherwise be explained by germline genetic aberrations. Identification of tumors early in the life of these patients, in particular in those with high risk of developing NF1-associated tumors, is important. For these patients, recommended follow-up via imaging modalities such as whole-body MRI and multidisciplinary NF clinics is required for timely and accurate diagnosis and management. It is hoped that continued understanding in the mechanisms of genetic aberrations in tumor development will lead to preventative and treatment methods for patients with NF1 and its associated tumors.

It is pertinent that all health care workers dealing with patients with NF are updated on the established and emerging genotype-phenotype correlations. High-throughput technology including NGS and WES is revolutionizing clinical research, leading to novel drug development and paving the path to precision medicine. We anticipate that improved genotyping and phenotyping methods combined with prudent approaches will help us understand the complexity of 
the gene, underlying molecular mechanisms, and heterogeneous phenotype of patients with NF.

\section{Acknowledgment}

The authors thank the Children's Tumor Foundation for support of the REiNS International Collaboration.

\section{Study Funding}

D.G.E. is supported by the All Manchester NIHR Biomedical Research Centre (IS-BRC-1215-20,007). C.B. is supported by NCI R21 CA208723, DOD W81XWH-16-0078, NCI R37 CA230400, and Burroughs Wellcome Career Award for Medical Scientists. C.O.H. is supported by funding from the Brain Tumour Research charity.

\section{Disclosure}

D.G.E. has received travel grants and consultancy fees from AstraZeneca. C.B. is a consultant for Depuy-Synthes. C.O.H., M.U., A.K., and D.M. report no disclosures relevant to the manuscript. Go to Neurology.org/N for full disclosures.

\section{Publication History}

Received by Neurology October 9, 2020. Accepted in final form March 19, 2021.

\section{Appendix Authors}

\begin{tabular}{lll}
\hline Name & Location & Contribution \\
\hline Chetan & Johns Hopkins University & $\begin{array}{l}\text { Major role in the acquisition } \\
\text { of data; analysis or }\end{array}$ \\
MD, PhD & Baltimore, MD & $\begin{array}{l}\text { interpretation of the data; } \\
\text { drafting or revising the } \\
\text { manuscript for intellectual } \\
\text { content }\end{array}$
\end{tabular}

\begin{tabular}{lll}
\hline $\begin{array}{l}\text { Meena } \\
\text { Upadhayaya, }\end{array}$ & Division Cancer and & Major role in the acquisition \\
PhD & UK & \\
& & $\begin{array}{l}\text { interpretation of the data; } \\
\text { drafting or revising the } \\
\text { manuscript for intellectual } \\
\text { content }\end{array}$
\end{tabular}

\begin{tabular}{ll}
\hline D. Gareth & Genomic Medicine, \\
Evans, MD & University of Manchester, \\
& UK
\end{tabular}

Major role in the acquisition of data; analysis or interpretation of the data; drafting or revising the manuscript for intellectual content

AeRang Kim, Center for Cancer and Major role in the acquisition MD, PhD Blood Disorders, ch National Hospital, Washington, DC

of data; analysis or interpretation of the data; drafting or revising the manuscript for intellectual content

\begin{tabular}{|c|c|c|}
\hline $\begin{array}{l}\text { Dimitrios } \\
\text { Mathios, MD }\end{array}$ & $\begin{array}{l}\text { Johns Hopkins University } \\
\text { School of Medicine, } \\
\text { Baltimore, MD }\end{array}$ & $\begin{array}{l}\text { Drafting or revising the } \\
\text { manuscript for intellectual } \\
\text { content }\end{array}$ \\
\hline $\begin{array}{l}\text { C. Oliver } \\
\text { Hanemann, } \\
\text { MD, PhD }\end{array}$ & $\begin{array}{l}\text { Faculty of Health, Medicine, } \\
\text { Dentistry and Health } \\
\text { Sciences, Institute of } \\
\text { Translational and Stratified } \\
\text { Medicine, University of } \\
\text { Plymouth, UK }\end{array}$ & $\begin{array}{l}\text { Design or conceptualization } \\
\text { of the study; major role in the } \\
\text { acquisition of data; analysis } \\
\text { or interpretation of the data; } \\
\text { drafting or revising the } \\
\text { manuscript for intellectual } \\
\text { content }\end{array}$ \\
\hline
\end{tabular}

\section{References}

1. Hanemann CO, Blakeley JO, Nunes FP, et al. Current status and recommendations for biomarkers and biobanking in neurofibromatosis. Neurology. 2016;87(7): S40-S48.

2. Stivaros SM, Stemmer-Rachamimov AO, Alston R, et al. Multiple synchronous sites of origin of vestibular schwannomas in neurofibromatosis type 2. J Med Genet. 2015; 52(8):557-562.

3. Dewan R, Pemov A, Kim HJ, et al. Evidence of polyclonality in neurofibromatosis type 2-associated multilobulated vestibular schwannomas. Neuro Oncol. 2015;17(4): 566-573.

4. Evans DG. Neurofibromatosis type 2 (NF2): a clinical and molecular review. Orphanet J Rare Dis. 2009;4:16.

5. Baser ME, Friedman JM, Aeschliman D, et al. Predictors of the risk of mortality in neurofibromatosis 2. Am J Hum Genet. 2002;71(4):715-723.

6. Hexter A, Jones A, Joe $\mathrm{H}$, et al. Clinical and molecular predictors of mortality in neurofibromatosis 2: a UK national analysis of 1192 patients. J Med Genet. 2015; 52(10):699-705.

7. Kalamarides M, Essayed W, Lejeune JP, et al. Spinal ependymomas in NF2: a surgical disease? J Neurooncol. 2018;136(3):605-611.

8. Evans DG, Howard E, Giblin C, et al. Birth incidence and prevalence of tumor-prone syndromes: estimates from a UK family genetic register service. Am J Med Genet A. 2010;152A(2):327-332.

9. Evans DG, Hartley CL, Smith PT, et al. Incidence of mosaicism in 1055 de novo NF2 cases: much higher than previous estimates with high utility of next-generation sequencing. Genet Med. 2020;22(1):53-59.

10. Parry DM, MacCollin MM, Kaiser-Kupfer MI, et al. Germ-line mutations in the neurofibromatosis 2 gene: correlations with disease severity and retinal abnormalities. Am J Hum Genet. 1996;59(3):529-539.

11. Baser ME, Kuramoto L, Woods R, et al. The location of constitutional neurofibromatosis 2 (NF2) splice site mutations is associated with the severity of NF2. J Med Genet. 2005;42(7):540-546.

12. Smith MJ, Higgs JE, Bowers NL, et al. Cranial meningiomas in 411 neurofibromatosis type 2 (NF2) patients with proven gene mutations: clear positional effect of mutations, but absence of female severity effect on age at onset. J Med Genet. 2011;48(4): 261-265

13. Halliday D, Emmanouil B, Pretorius P, et al. Genetic Severity Score predicts clinical phenotype in NF2. J Med Genet. 2017;54(10):657-664.

14. Ruttledge MH, Andermann AA, Phelan CM, et al. Type of mutation in the neurofibromatosis type 2 gene (NF2) frequently determines severity of disease. Am J Hum Genet. 1996;59(2):331-342.

15. Emmanouil B, Houston R, May A, et al. Progression of hearing loss in neurofibromatosis type 2 according to genetic severity. Laryngoscope. 2019;129(4):974-980.

16. Upadhyaya M, Huson SM, Davies M, et al. An absence of cutaneous neurofibromas associated with a 3-bp in-frame deletion in exon 17 of the NF1 gene (c.2970-2972 delAAT): evidence of a clinically significant NF1 genotype-phenotype correlation. Am J Hum Genet. 2007;80(1):140-151.

17. Koczkowska M, Chen Y, Callens T, et al. Genotype-phenotype correlation in NF1: evidence for a more severe phenotype associated with missense mutations affecting NF1 codons 844-848. Am J Hum Genet. 2018;102(1):69-87.

18. van Minkelen R, van Bever Y, Kromosoeto JN, et al. A clinical and genetic overview of 18 years neurofibromatosis type 1 molecular diagnostics in The Netherlands. Clin Genet. 2014;85(4):318-327.

19. Brems H, Chmara M, Sahbatou M, et al. Germline loss-of-function mutations in SPRED1 cause a neurofibromatosis 1-like phenotype. Nat Genet. 2007;39(9): $1120-1126$.

20. Pinna V, Lanari V, Daniele P, et al. p.Arg1809Cys substitution in neurofibromin is associated with a distinctive NF1 phenotype without neurofibromas. Eur J Hum Genet. 2015;23(8):1068-1071.

21. Rojnueangnit K, Xie J, Gomes A, et al. High incidence of Noonan syndrome features including short stature and pulmonic stenosis in patients carrying NF1 missense mutations affecting p.Arg1809: genotype-phenotype correlation. Hum Mutat. 2015; 36(11):1052-1063.

22. Kehrer-Sawatzki H, Mautner VF, Cooper DN. Emerging genotype-phenotype relationships in patients with large NF1 deletions. Hum Genet. 2017;136(4):349-376.

23. Kehrer-Sawatzki H, Kluwe L, Sandig C, et al. High frequency of mosaicism among patients with neurofibromatosis type 1 (NF1) with microdeletions caused by somatic recombination of the JJAZ1 gene. Am J Hum Genet. 2004;75(3):410-423.

24. Upadhyaya M, Ruggieri M, Maynard J, et al. Gross deletions of the neurofibromatosis type 1 (NF1) gene are predominantly of maternal origin and commonly associated with a learning disability, dysmorphic features and developmental delay. Hum Genet. 1998;102(5):591-597.

25. Pasmant E, Sabbagh A, Spurlock G, et al. NF1 microdeletions in neurofibromatosis type 1: from genotype to phenotype. Hum Mutat. 2010;31(6):E1506-E1518.

26. Douglas J, Cilliers D, Coleman K, et al. Mutations in RNF135, a gene within the NF1 microdeletion region, cause phenotypic abnormalities including overgrowth. Nat Genet. 2007;39(8):963-965.

27. Messiaen L, Vogt J, Bengesser K, et al. Mosaic type-1 NF1 microdeletions as a cause of both generalized and segmental neurofibromatosis type-1 (NF1). Hum Mutat. 2011; $32(2): 213-219$

28. Kluwe L, Tatagiba M, Funsterer C, Mautner VF. NF1 mutations and clinical spectrum in patients with spinal neurofibromas. J Med Genet. 2003;40(5):368-371.

29. Pulst SM, Riccardi VM, Fain P, Korenberg JR. Familial spinal neurofibromatosis: clinical and DNA linkage analysis. Neurology. 1991;41(12):1923-1927. 
30. Upadhyaya M, Spurlock G, Kluwe L, et al. The spectrum of somatic and germline NF1 mutations in NF1 patients with spinal neurofibromas. Neurogenetics. 2009;10(3):251-263.

31. Burkitt Wright EM, Sach E, Sharif S, et al. Can the diagnosis of NF1 be excluded clinically? A lack of pigmentary findings in families with spinal neurofibromatosis demonstrates a limitation of clinical diagnosis. J Med Genet. 2013;50(9):606-613.

32. Ruggieri M, Polizzi A, Spalice A, et al. The natural history of spinal neurofibromatosis: a critical review of clinical and genetic features. Clin Genet. 2015;87(5):401-410.

33. Frayling IM, Mautner VF, van Minkelen R, et al. Breast cancer risk in neurofibromatosis type 1 is a function of the type of NF1 gene mutation: a new genotypephenotype correlation. J Med Genet. 2019;56(4):209-219.

34. Dodd RD, Lee CL, Overton T, et al. NF1(+/-) hematopoietic cells accelerate malignant peripheral nerve sheath tumor development without altering chemotherapy response. Cancer Res. 2017;77(16):4486-4497.

35. Zhang M, Wang Y, Jones S, et al. Somatic mutations of SUZ12 in malignant peripheral nerve sheath tumors. Nat Genet. 2014;46(11):1170-1172.

36. Lee W, Teckie S, Wiesner T, et al. PRC2 is recurrently inactivated through EED or SUZ12 loss in malignant peripheral nerve sheath tumors. Nat Genet. 2014;46(11):1227-1232.

37. Sohier P, Luscan A, Lloyd A, et al. Confirmation of mutation landscape of NF1associated malignant peripheral nerve sheath tumors. Genes Chromosomes Cancer. 2017;56(5):421-426.

38. Riccardi VM. The genetic predisposition to and histogenesis of neurofibromas and neurofibrosarcoma in neurofibromatosis type 1. Neurosurg Focus. 2007;22(6):E3.

39. Pekmezci M, Reuss DE, Hirbe AC, et al. Morphologic and immunohistochemical features of malignant peripheral nerve sheath tumors and cellular schwannomas. Mod Pathol. 2015;28(2):187-200.

40. Rodriguez FJ, Folpe AL, Giannini C, Perry A. Pathology of peripheral nerve sheath tumors: diagnostic overview and update on selected diagnostic problems. Acta Neuropathol. 2012;123(3):295-319.
41. Miettinen MM, Antonescu CR, Fletcher CDM, et al. Histopathologic evaluation of atypical neurofibromatous tumors and their transformation into malignant peripheral nerve sheath tumor in patients with neurofibromatosis 1 : a consensus overview. Hum Pathol. 2017;67:1-10.

42. Pemov A, Hansen NF, Sindiri S, et al. Low mutation burden and frequent loss of CDKN2A/B and SMARCA2, but not PRC2, define pre-malignant neurofibromatosis type 1-associated atypical neurofibromas. Neuro Oncol. 2019;21(8):981-992.

43. De Raedt T, Beert E, Pasmant E, et al. PRC2 loss amplifies Ras-driven transcription and confers sensitivity to BRD4-based therapies. Nature. 2014;514(7521):247-251.

44. Hirbe AC, Dahiya S, Miller CA, et al. Whole exome sequencing reveals the order of genetic changes during malignant transformation and metastasis in a single patient with NF1-plexiform neurofibroma. Clin Cancer Res. 2015;21(18):4201-4211.

45. Nix JS, Haffner MC, Ahsan S, et al. Malignant peripheral nerve sheath tumors show decreased global DNA methylation. I Neuropathol Exp Neurol. 2018;77:958-963.

46. Danielsen SA, Lind GE, Kolberg M, et al. Methylated RASSF1A in malignant peripheral nerve sheath tumors identifies neurofibromatosis type 1 patients with inferior prognosis. Neuro Oncol. 2015;17(1):63-69.

47. Feber A, Wilson GA, Zhang L, et al. Comparative methylome analysis of benign and malignant peripheral nerve sheath tumors. Genome Res. 2011;21(4):515-524.

48. Baylin SB, Ohm JE. Epigenetic gene silencing in cancer: a mechanism for early oncogenic pathway addiction? Nat Rev Cancer. 2006;6(2):107-116.

49. Lu HC, Eulo V, Apicelli AJ, et al. Aberrant ATRX protein expression is associated with poor overall survival in NF1-MPNST. Oncotarget. 2018;9(33):23018-23028.

50. Firth HV, Richards SM, Bevan AP, et al. DECIPHER: Database of chromosomal imbalance and phenotype in humans using ensembl resources. Am J Hum Genet. 2009 84(4):524-533.

51. Kohler S, Vasilevsky NA, Engelstad M, et al. The Human Phenotype Ontology in 2017. Nucleic Acids Res. 2017;45(D1):D865-D876. 


\section{Neurology}

\section{Genotype-Phenotype Correlations in Neurofibromatosis and Their Potential Clinical Use}

Chetan Bettegowda, Meena Upadhayaya, D. Gareth Evans, et al. Neurology 2021;97;S91-S98 Published Online before print July 6, 2021

DOI 10.1212/WNL.0000000000012436

\section{This information is current as of July 6, 2021}

\section{Updated Information \& Services}

References

Citations

Subspecialty Collections

Permissions \& Licensing

Reprints including high resolution figures, can be found at: http://n.neurology.org/content/97/7_Supplement_1/S91.full

This article cites 51 articles, 13 of which you can access for free at: http://n.neurology.org/content/97/7_Supplement_1/S91.full\#ref-list-1

This article has been cited by 1 HighWire-hosted articles: http://n.neurology.org/content/97/7_Supplement_1/S91.full\#\#otherartic les

This article, along with others on similar topics, appears in the following collection(s):

\section{All Genetics}

http://n.neurology.org/cgi/collection/all_genetics

Nerve tumor

http://n.neurology.org/cgi/collection/nerve_tumor

Neurofibromatosis

http://n.neurology.org/cgi/collection/neurofibromatosis

Outcome research

http://n.neurology.org/cgi/collection/outcome_research

Primary brain tumor

http://n.neurology.org/cgi/collection/primary_brain_tumor

Information about reproducing this article in parts (figures,tables) or in its entirety can be found online at:

http://www.neurology.org/about/about_the_journal\#permissions

Information about ordering reprints can be found online:

http://n.neurology.org/subscribers/advertise

Neurology ${ }^{\circledR}$ is the official journal of the American Academy of Neurology. Published continuously since 1951, it is now a weekly with 48 issues per year. Copyright (O) 2021 American Academy of Neurology. All rights reserved. Print ISSN: 0028-3878. Online ISSN: 1526-632X.

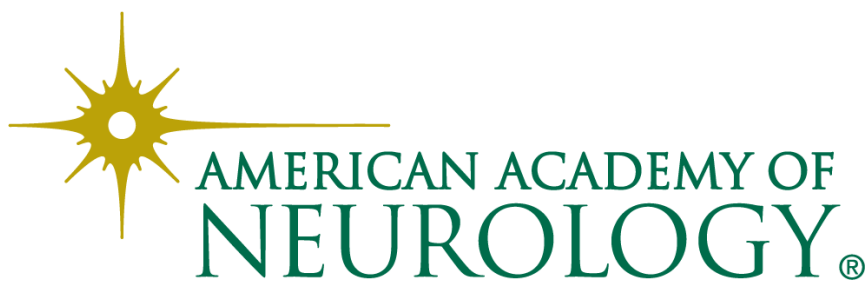

